\title{
A knowledge-based engineering approach for supporting railway manufacturers from the tender notice to the designing phase
}

\author{
G. Di Gironimo ${ }^{1}$, A. Lanzotti $^{1}$, R. Mozzillo ${ }^{1}$, F. Peluso ${ }^{1}$, \\ G. Calvano ${ }^{2}$, S. Puzelli ${ }^{2} \&$ P. Verdosci ${ }^{2}$ \\ ${ }^{1}$ University of Naples Federico II, DII, IDEAS Lab, Italy \\ ${ }^{2}$ AnsaldoBreda SpA., Italy
}

\begin{abstract}
This paper deals with the development of a knowledge-based engineering (KBE) approach able to support railway manufacturers in their assessments on the convenience of participating in competitive tendering and, subsequently, in the offer definition and in the designing phase. The proposed approach is based on a Decision Support System (DSS) that allows an analysis, called Adopt-Adapt-Innovate (AAI), to be made, which helps the company in the search of its products that best suit the requirements of new bids. Digital pattern techniques, configuration design methods and parametric modeling are the tools proposed to optimize the process that starts with the tender notice, passes through the offer definition and ends with the design. The paper describes the railway market logics, the proposed methodology and the first obtained results.

Keywords: knowledge-based engineering, decision support system, digital pattern, parametric modeling.
\end{abstract}

\section{Introduction}

The economic downturn and the increasingly demanding markets are forcing companies to reduce wrong and unprofitable investments, and search for means to decrease time and costs for new product development while satisfying customer requirements. In the design domain, one of the technologies that can support the decision-making phase and rapid, modular design is knowledgebased engineering (KBE). Many definitions of KBE exist in literature [1-3]. All 
of these definitions can be synthesized as follows. KBE is the use of advanced software techniques, and the study of innovative methodologies, to capture and re-use product and process engineering knowledge in an integrated way. Its fundamentals are clearly reported in [4]. As the definitions state, one of the hallmarks of the KBE approach is to automate repetitive, non-creative design tasks. As exposed in [5] not only does automation permit significant time and cost savings, it also frees up time for creativity, which allows exploration of a larger part of the design envelope (Figure 1).

Successful efforts to implement and benefit from KBE have been made in various manufacturing sectors.

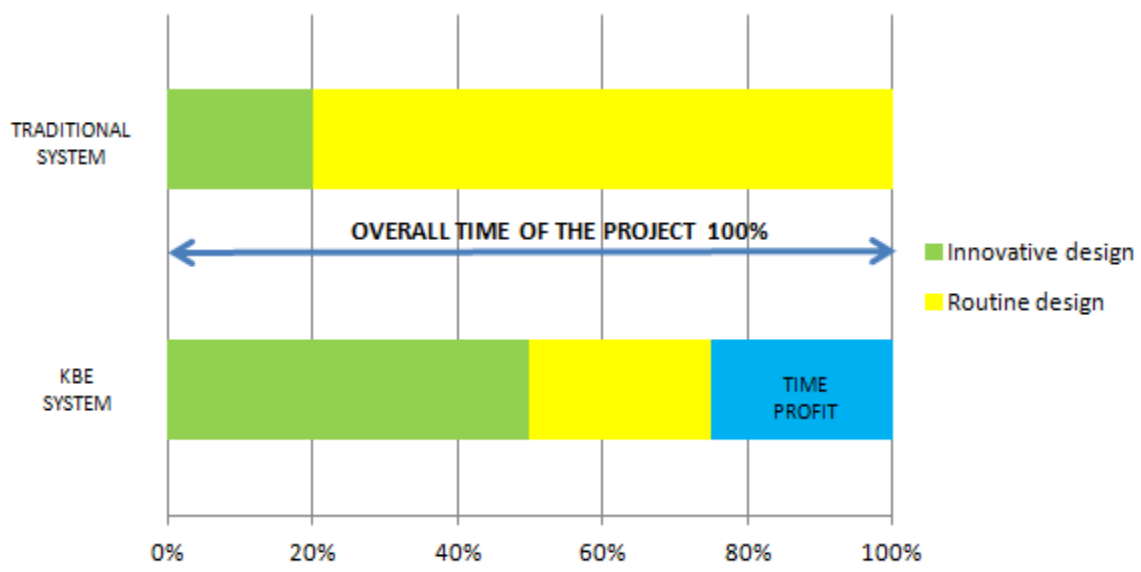

Figure 1: Influence of KBE usage on time of main design tasks.

For instance, in [6] is described the development of a multidisciplinary system that is able to rapidly generate car designs. In the aerospace domain, there is research [7] dealing with a system that automates design effort for blended wing bodies; [8] describing an application for the design of low pressure turbines. KBE systems are also developed for the design of manufacturing tools and processes $[9,10]$. By contrast, there are only few researches and applications in the railway manufacturing industry [11].

This paper derives from a research born with the scope of designing and implementing a KBE system for supporting a firm acting in the railway market. The activities have been conducted in collaboration with a train manufacturer company, AnsaldoBreda SpA, a Finmeccanica Company.

\section{The railway market}

Different from other manufacturing sectors - i.e. the automotive industry - in the railway market the producer of a train is not the actor that decides the features of the vehicle it is going to produce. Indeed, this role lies with the customer and the 
regulatory rules of the country in which the vehicle is going to serve. In detail, the commissioning body (the customer) issues an invitation to tender (ITT) that consists, inter alia, of the train-requirements list. These requirements are organized into the technical specifications document.

As illustrated in Figure 2, the process begins with the ITT and continues with the bidding process. The company makes technical and economical feasibility analyses, and chooses, in this case, to take part in the tender (bid/no bid choice); the tender offer then follows. Finally, in the case of a winning bid, there is the design and the production of the train.

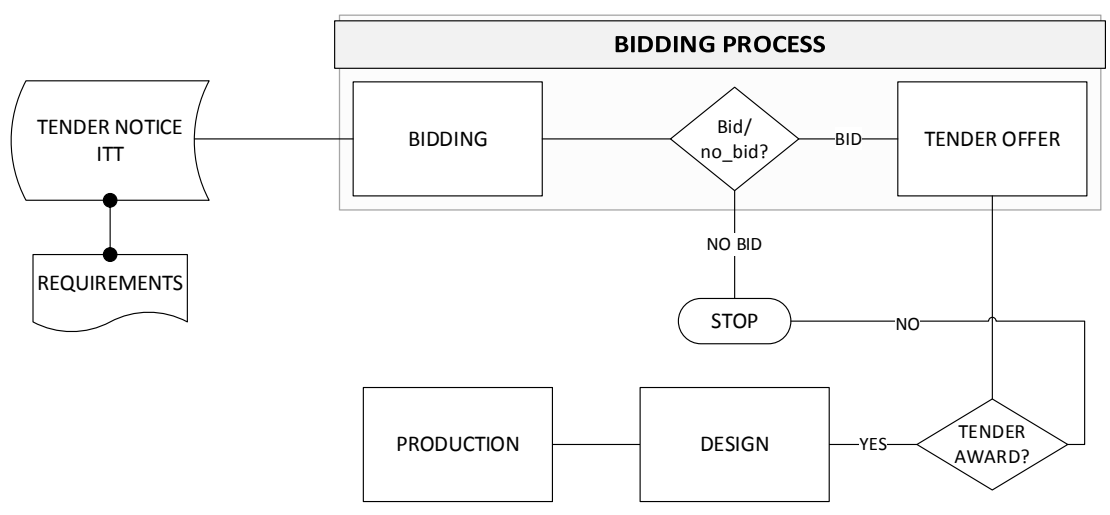

Figure 2: $\quad$ The flow chart from the ITT to the production phase.

\section{The methodology: knowledge capture, retention and re-use}

In medium and large companies, a simple and efficient re-use of the knowledge base can be difficult because of the huge amount of data. An effective data management system is mandatory if the company wants to take advantage of this asset. For a railway manufacturer, as mentioned above, a new project begins with the bid/no-bid choice and possibly continues with a competitive and profitable tender offer. Typically, these tasks must be done in a fixed and short time frame and the constraints imposed by internal communication may hinder participation in the competition. In the worst case, there is the risk of participating with hasty and inaccurate analyses. Searching for a way to support these critical phases, the authors have researched a new methodology.

\subsection{The Decision Support System}

First, a Decision Support System (DSS) has been designed into the PLM system (which is a classifier of the whole company knowledge in terms of designed/produced trains, subsystems and/or components) with the aim of realizing a "catalog" of standard products. This catalog can be examined in 
order to search for the component part of the vehicle that is "similar" to the one requested by the customer.

The data structure of the DSS consists of three classes:

- At the first level of the structure there is the class vehicle: the root object of the Product Breakdown Structure (PBS); the family to which the vehicle belongs (i.e. high-speed train, automatic light metro, metro-tram) is an attribute of this class.

- At the second level, there are the subsystems: mock objects that identify a function of a train or a typical element of the organization of a train or, finally, a typical element of the assembly process of the vehicle (i.e. vehicle body, power system, pneumatic/hydraulic equipment).

- At the last level, there are the components: items of the E-BOM (Engineering Bill of Materials) characterized by classification attributes (i.e. bogie, traction motor, pantograph).

The classification has been made with attributes of various genres: master data; normative data; technical data; performances data; mechanical characteristics; environmental characteristics; electrical data; other useful data.

Furthermore, every component has a link to the corresponding Configuration Item (CI) stored in the PDM system, containing all the files and the documents related to it.

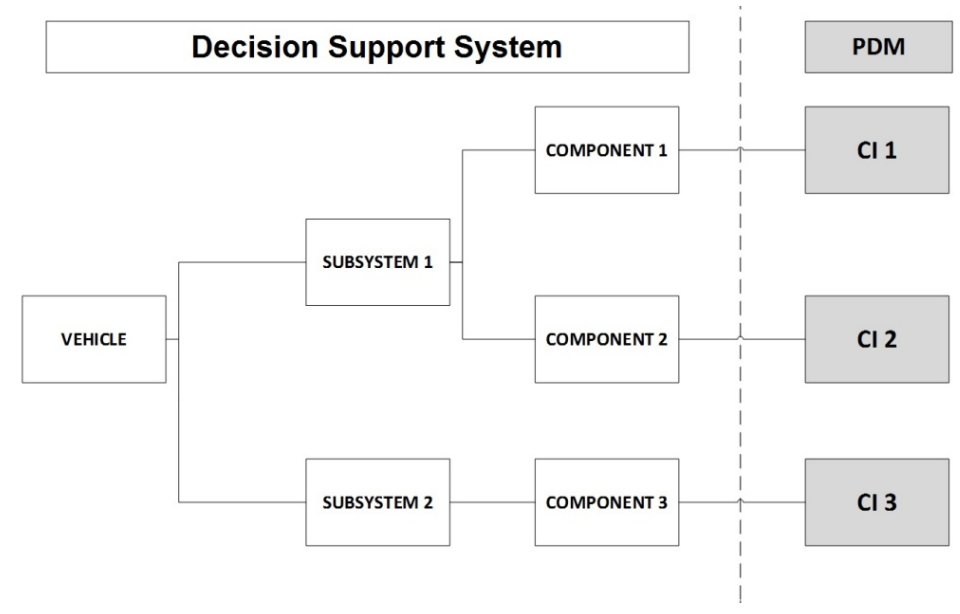

Figure 3: The three-level structure of the DSS and the link with the PDM.

The class subsystem combines the functions of the vehicle as configured at the second level of the standard PBS, carried out on the basis of the structure of product groups as defined by 'EN 15380-2 Product Group Code Letters for Railway Applications - Designation system for railway vehicles - Part 2: Product groups'. In Figure 4, the three-layer data model implemented into the PLM system is shown. 


\subsection{Adopt-Adapt-Innovate analysis}

The workflow that, starting from the ITT, allows a vehicle/component configuration to be obtained, which is identified according to the PBS functions and to the customer needs, is:

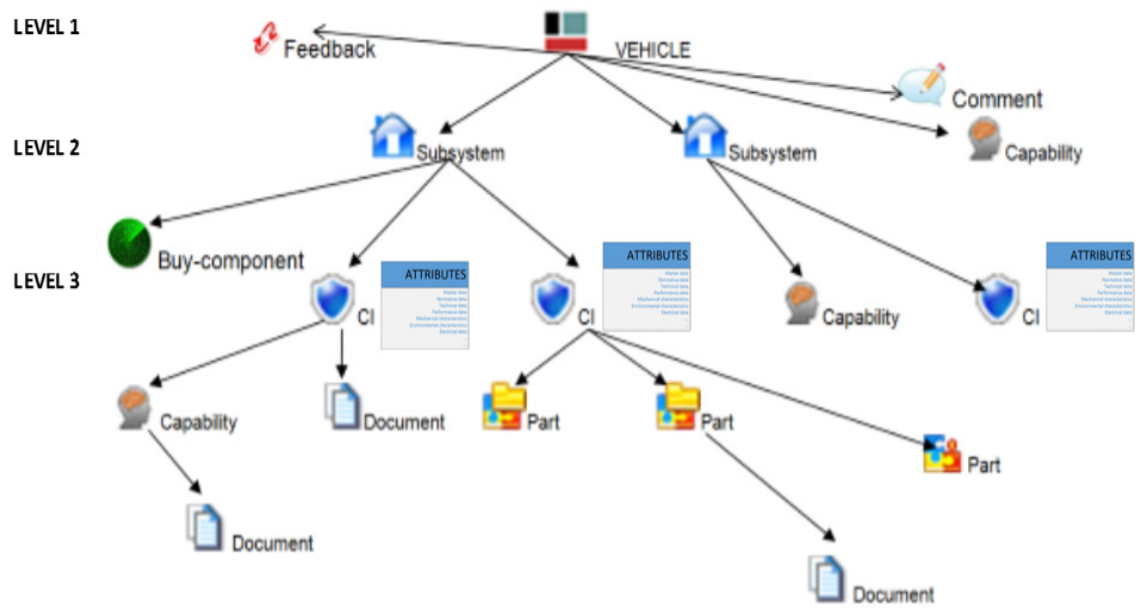

Figure 4: The data model of the product catalog.

1. A set of requirements/features (established at the level of the components) is constructed from the technical specifications document.

2. A search is performed, into the DSS, with this set of requirements/features as input, comparing what the customer wants and what is classified in the catalog.

3. The Adopt-Adapt-Innovate (AAI) analysis starts: the system returns the details of the comparison and a series of synthetic indexes of correspondence.

4. A first-proposal of vehicle/component configuration, identified according to the PBS functions and to the customer's needs, is automatically generated by the PLM system. This configuration may have some empty data fields, meaning that there is no $\mathrm{CI}$ in the catalog that matches the technical specifications.

5. A context of product is generated, where it is possible to manage the new product and make technical and economical analyses. This is the end of the AAI.

6. The adapting phase through the use of template CAD products (see the next paragraph) starts.

7. When the adaptation is not possible, an innovation may be necessary.

The AAI analysis, a pivotal point of the proposed methodology, works as follows. CIs referred to as "adoptable", that is, whose attributes correspond to $100 \%$ of the requirements, are automatically inserted into the configuration of the new vehicle; CIs referred to as "adaptable", that is, to be modified so that 
their features match the requirements, are copied onto a new CI for the subsequent changes/adaptations according to the technical specifications. Eventually, if for some item of the E-BOM the KBE system does not find any CI which matches the requirements, that is, the matching percentage is too low, the corresponding leaf node (at the third level of the PBS) is empty, meaning that an innovation is necessary. Thus, at the end of the AAI, the system returns a firstproposal vehicle configuration (Figure 5).

LEVEL 1

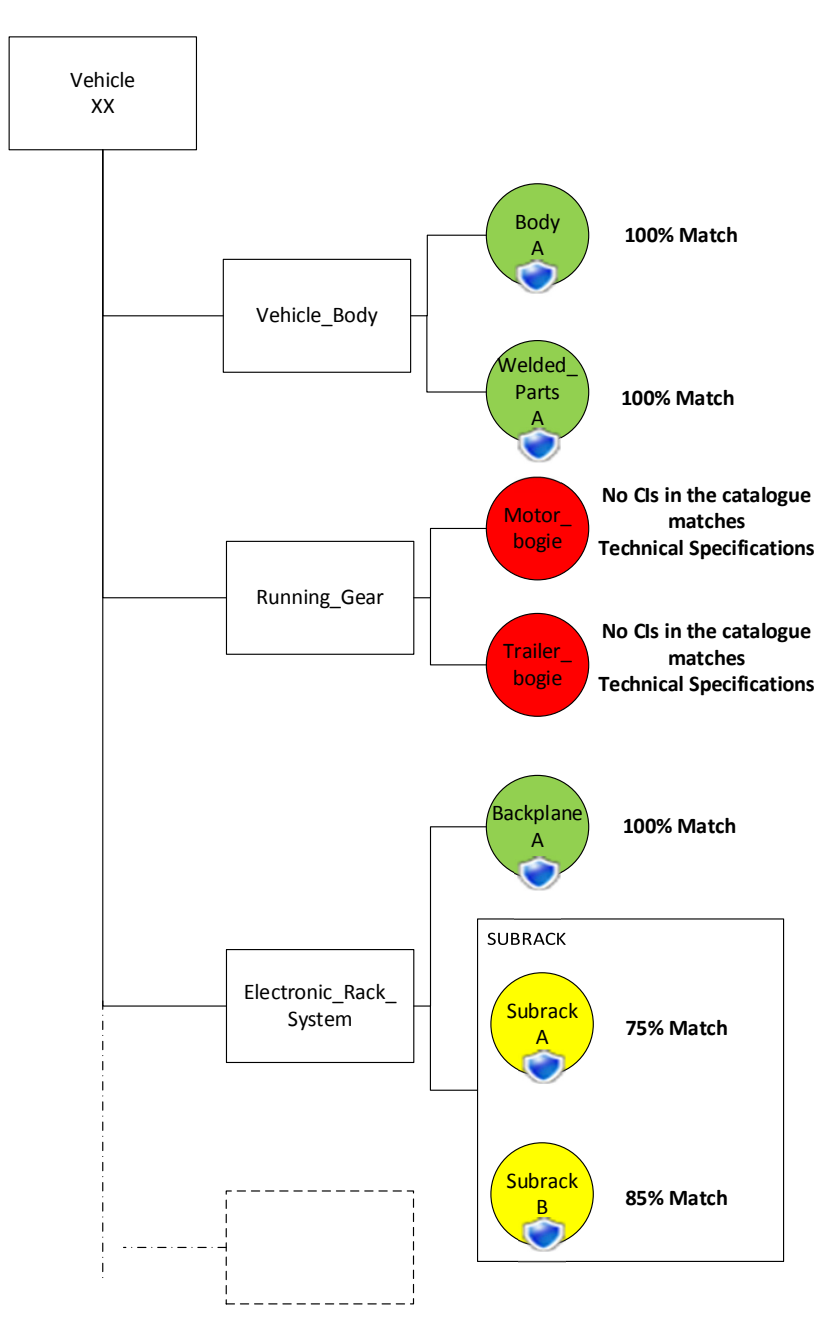

ADOPTING

ADAPTING

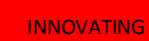

Figure 5: Simplified example of a first proposal vehicle configuration identified according to the PBS functions and to the customer needs. 
As shown in Figure 5, it may happen that a leaf node has two or more adaptable CIs. In this case, the system engineer can choose, between the proposed CIs, the most suitable one on the basis of personal technical considerations.

At the end of the AAI analysis, the company makes the bid/no bid choice.

\subsection{Adapting phase through the template product}

The template product acts in the designing phase, once the tender has been won. Normally, the redesign of a product may be time consuming and can require repetitive actions. The methodology proposed in this paper suggests the use of a template product, that is a $3 \mathrm{D}$ mathematical model of a component that encloses or contains all the features of a whole family of products, conditional expressions (if-then-else constructs) and some technical constraints information. Starting from the template product the user can configure new products with similar but different characteristics.

\section{The template product case study: the rack of the traction control unit}

As clearly illustrated in [12], "the challenge of reducing designing time [...], especially in the context of large companies, encourages the use of methods and tools aimed to support designing activities and to re-use the company knowhow". Inspired by the design parameterization methodology, that is: "to automatically complete new parts design through changing the parameter value of the existing design results" [13], a template CAD product has been developed with the focus on speeding up the design activities. In particular, the authors concentrated on the development of a methodology and a tool that:

- contributes to reducing the time to market of products;

- is able to interact with the PDM/PLM system and contribute to its population;

- $\quad$ is interactive.

The template product case study is born from the consideration that a commercial CAD systems' ability of supporting the parametric modeling of parts is high and well known, but the parametric design of assembly models is not well sustained [14]. The first assembly modeled in a template way is the rack of the traction control unit (TCU). This is a product that does not have a high level of complexity, and whose components have a high level of standardization. These features are well suited to a case study. The developed template rack has a high-parametric structure; the user interacts with it through the independent variables that are related to all the other dependent parameters. By making a suitable choice of these variables the designer quickly comes to the definition of the product, all of its features and all the technical documentation required for its production: in fact, the annotations have been included in the $3 \mathrm{D}$ model. In this way, the user automatically generates the geometry of the assembly complete 
with dimensions, tolerances and all the information necessary for the production of the component.

The rack consists of several sub-assemblies and they were modeled separately and then inserted into an overall architecture that allows the user to manage the entire variability of the rack family.

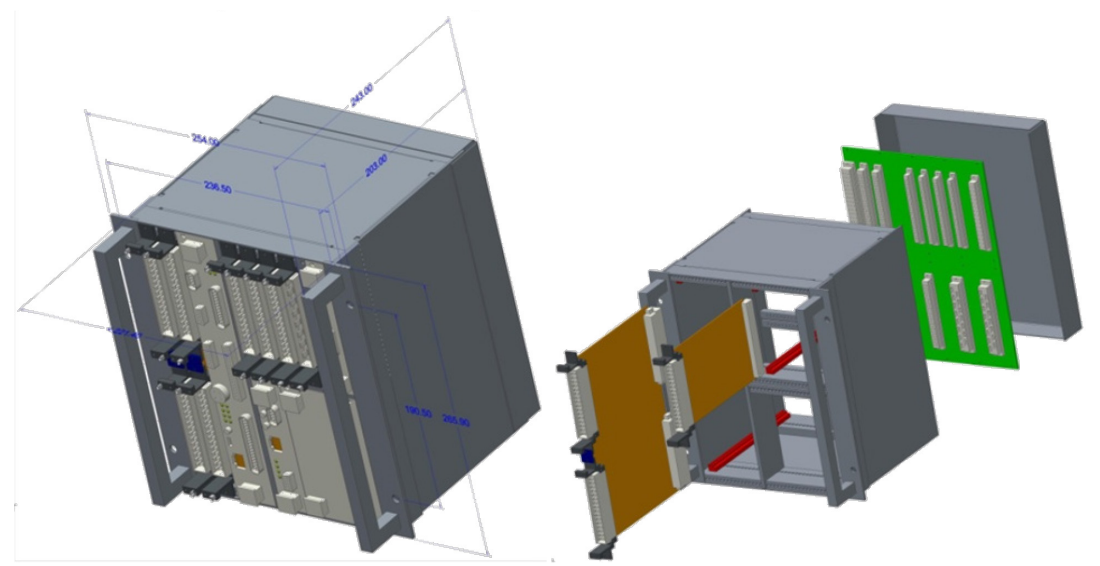

Figure 6: An instance of the template rack complete with the 3D annotations and an exploded view.

The activity started with a detailed analysis of the assembly to be modeled aiming at identifying:

- the sub-components and the features to model;

- the independent parameters that control the variability of the subcomponents and the assembly;

- the functional relationships between the sub-components.

The template has been completely parameterized. In this way, the user can get any configuration of the rack by simply typing the input data from the keyboard, without taking any action on the 3D model. It should be noted that the parameterization affects not only geometrical and dimensional parameters, but also binary parameters or strings, which can manage the presence or absence of features and parts.

The CAD tool used for modeling the template rack is Creo2.0 by PTC. In detail, to obtain the template product, two tools of the Creo2.0 suite have been used:

- the pro/program module;

- the family table tool.

Pro/program is a programming module. It allows users to manage and edit the strings of code that Creo2.0 generates when modeling the various parts/components. In fact, each part or assembly is associated with some lines of code and, by editing the code through pro/program in an appropriate way; it is possible to program the model and obtain a conditional modeling. One of the possibilities provided by this tool is to manage the parameters via questions that are asked of the user. Using this approach, each configuration of the product is 
identified by a set of values for the independent parameters chosen. In this way, the designer has only to define the set of values and does not have to worry about the modeling and the creation of documentation.

The family table tool lets you create multiple versions of a part or an assembly, changing the values of parameters such as dimensions and managing the presence of features and components. This tool gives the designer the opportunity to populate the product catalog after he/she has defined the set of parameters. Within the template has been implemented the option of saving the set of input parameters by creating an instance of the family table.

The rack of the TCU is composed of the structural unit and the inserted electronic boards. The boards allow the functions of the vehicle related to the traction to be managed. Thus, the assembly rack has been broken down into its sub-components: the sub-rack; the backplane; the electronic board; the board rail.

The sub-rack is the unit intended to house the printed circuit boards. The backplane, which is a plate for housing the rear connectors, is mounted inside the sub-rack. The boards are mounted on the sub-rack with the appropriate board rails.

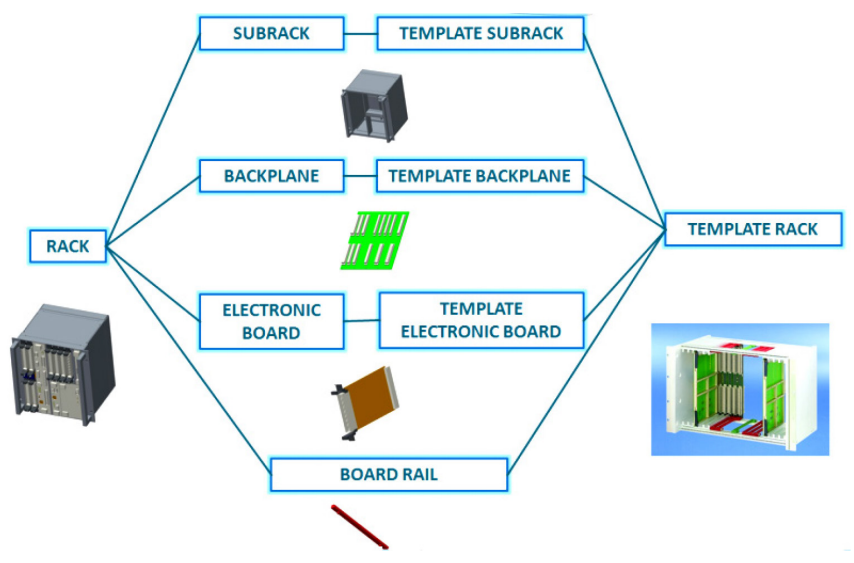

Figure 7: The rack and its subcomponents.

The template model handles the geometric variability of the sub-rack, the backplane and the boards. It also allows the boards in the assembly to be positioned. The flow chart for obtaining the desired rack is reported in Figure 8.

1. The user chooses the sub-rack through a series of questions/answers.

2. The system queries the catalog to check whether the required backplane already exists and, if not, generates a new backplane on the base of the input.

3. The system queries the library to check whether the required type of board is already present in the catalog and, if not, a new board type is generated based on the input.

4. The user chooses the number of boards and their position. 
The input data are put into the model via the user interface, passing by a series of questions through a sequence of pop-up windows (see Figure 9). By simply entering these data the user gets the whole geometric model, complete with $3 \mathrm{D}$ annotations and the technical documentation.

So the modeling phase and production of technical documentation is reduced to the simple choice of the set of input parameters. Everything else is handled entirely and automatically by the template CAD file.

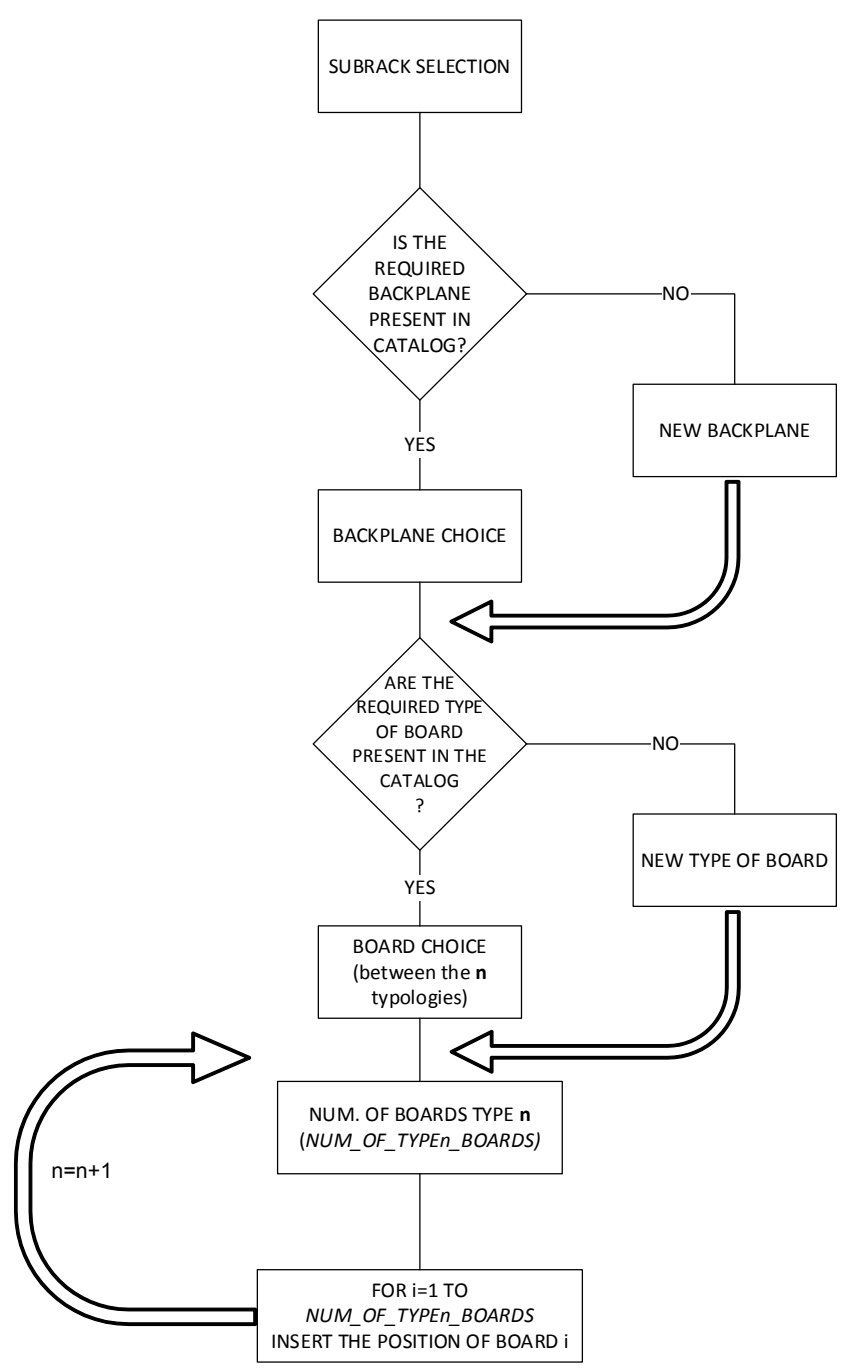

Figure 8: The flow chart for assembling the rack. 


\section{Conclusions, results and future works}

The aim of the research has been to study a methodology and implement a system that supports a company acting in the railway market in the evaluation of its existing products that best suit the requirements of new bids. The proposed approach and system allow the company to carry out technical and economic evaluations in order to assess the use of its technical know-how within new projects. The use of the product catalog and the AAI analysis dramatically reduce the duration of the bidding process. Before the use of the new methodology, the time from the tender notice to the tender offer was about 60 days. As a result of the first simulations, it can be said that the time has now been halved.

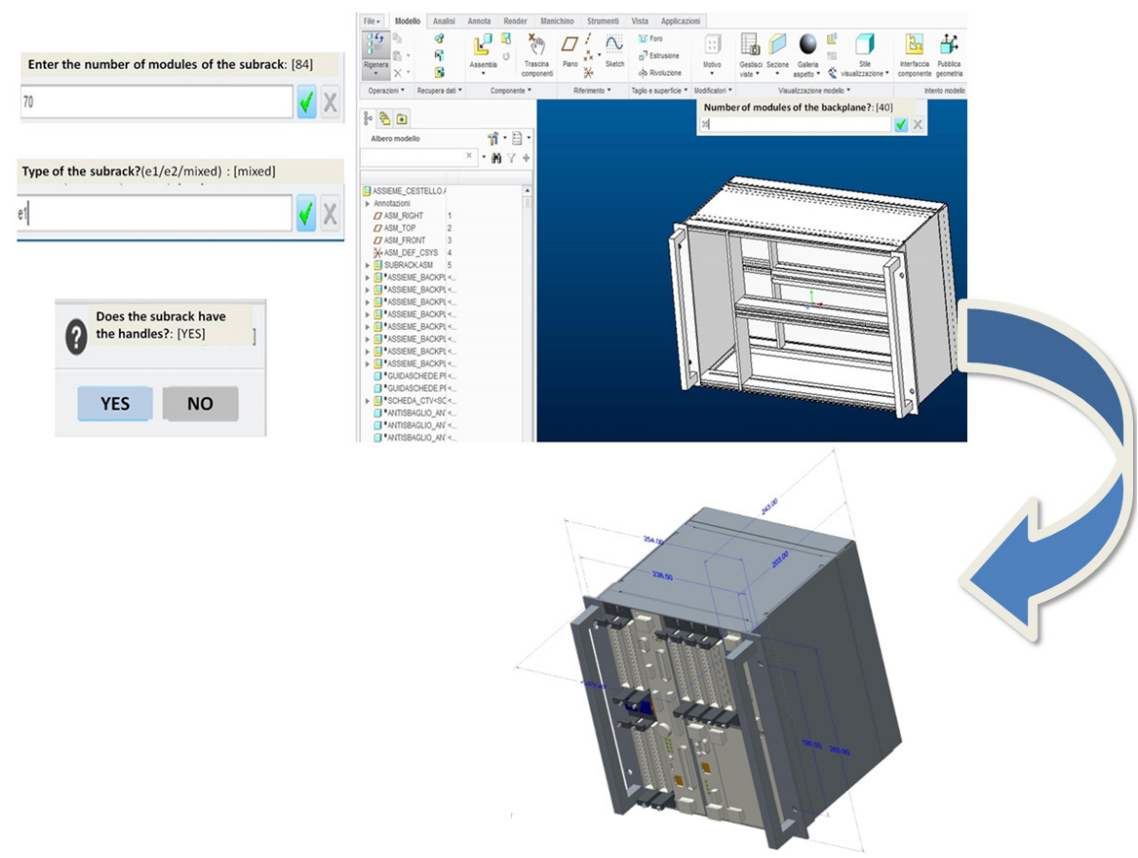

Figure 9: Examples of questions for the data-entering phase. After this phase, the desired rack is automatically generated.

In addition, the use of this objective and rational methodology reduces, at the minimum, the risk of participating in a not technically sustainable or economically convenient competitive tendering.

The other strand of the research has been the introduction of the template. The study conducted on the rack of the TCU has demonstrated that the time required for the designing phase can be, for components of average complexity, more than halved, bringing obvious economic and time benefits. Certainly, the creation of a template $\mathrm{CAD}$ file takes time and the entry phase of data can be 
heavy when the input values are numerous. On the other hand, the model composes by itself at the end of the parameter-entering phase. Future objectives are researching on more powerful template CAD files, testing the tool on more complex products, implementing techniques which may make the tool more interactive.

\section{Acknowledgements}

The work presented in this paper has been partially funded by MIUR (Italian Ministry of University and Research) and Campania regional authority within, respectively, the PON project "Digital Pattern" and the Network of Excellence framework "INSIST". Authors thank AnsaldoBreda SpA for the case study and PM Technology Consulting for the technical support.

\section{References}

[1] Baxter, D., Gao, J., Case, K., Harding, J., Young, B., Cochrane, S., Dani, S., An engineering design knowledge reuse methodology using process modelling, Research in Engineering Design, 18, pp. 37-48, 2007.

[2] Stokes, M., Ed. Managing Engineering Knowledge-MOKA: Methodology for Knowledge Based Engineering Applications, ASME Press, 2001.

[3] Cooper, D.J., La Rocca, G., Knowledge-based techniques for developing engineering applications in the 21st century, Proceedings of the 7th AIAA Aviation Technology, Integration and Operations Conference, Belfast, 2007.

[4] Sandberg, M., Technical Report - Knowledge Based Engineering: In Product Development, Lulea University of Technology, 2003.

[5] Skarka, W., Application of MOKA methodology in generative model creation using CATIA, Engineering Applications of Artificial Intelligence, 20, pp. 677-690, 2007.

[6] Chapman, C.B., Pinfold, M., The application of a knowledge based engineering approach to the rapid design and analysis of an automotive structure, Advances in Engineering Software, 32, pp. 903-912, 2001.

[7] La Rocca, G., Van Tooren, M.J.L., Enabling distributed multi-disciplinary design of complex products: a knowledge based engineering approach, Journal of Design Research, 5, pp. 333-352, 2007.

[8] Corallo, A., Laubacher, R., Margherita, A., Turrisi, G., Enhancing product development through knowledge-based engineering (KBE): a case study in the aerospace industry, Journal of Manufacturing Technology Management, 20, pp. 1070-1083, 2009.

[9] Kulon, J., Mynors, D. J., Broomhead, P., A knowledge-based engineering design tool for metal forging, Journal of Materials Processing Technology, 177, pp. 331-335, 2006.

[10] Van der Laan, A.H., Curran, R., Van Tooren, M.J.L., Ritchie, C., Integration of friction stir welding into a multidisciplinary aerospace design framework, Aeronautical Journal, 110, pp. 759-766, 2006. 
[11] Di Gironimo, G., Patalano, S., Re-Design of a Railway Locomotive in Virtual Environment for Ergonomic Requirements, International Journal on Interactive Design and Manufacturing, 2(1), pp. 47-57, 2008.

[12] Patalano, S., Vitolo, F. and Lanzotti, A., A Graph-based Software Tool for the CAD Modeling of Mechanical Assemblies, Proceedings of the International Conference on Computer Graphics Theory and Applications and International Conference on Information Visualization Theory and Applications GRAPP 2013, Barcelona, pp. 60-69, 2013.

[13] Xu, Y., Hu, L., Zeng, W., Jin, B., Web-service-based parametric design reuse for parts, International Journal of Advanced Manufacturing Technology, 46, pp. 423-429, 2010.

[14] Myung, S., Han, S., Knowledge-based parametric design of mechanical products based on configuration design method, Expert Systems with Applications, 21, pp. 99-107, 2001. 